\title{
Preoperative platelet to lymphocyte ratio is a valuable prognostic biomarker in patients with colorectal cancer
}

\author{
Jie You ${ }^{1,2, *}$, Gui-Qi Zhu ${ }^{3,4, *}$, Linka Xie ${ }^{5, *}$, Wen-Yue Liu', Liang Shi7, Ou-Chen Wang ${ }^{1}$, \\ Zong-Hai Huang ${ }^{2}$, Martin Braddock ${ }^{8}$, Gui-Long Guo', Ming-Hua Zheng ${ }^{3,9}$ \\ ${ }^{1}$ Department of Oncological Surgery, The First Affiliated Hospital of Wenzhou Medical University, Wenzhou 325000, China \\ ${ }^{2}$ Department of General Surgery, Zhujiang Hospital, Southern Medical University, Guangzhou 510282, China \\ ${ }^{3}$ Department of Infection and Liver Diseases, Liver Research Center, The First Affiliated Hospital of Wenzhou Medical \\ University, Wenzhou 325000, China \\ ${ }^{4}$ School of the First Clinical Medical Sciences, Wenzhou Medical University, Wenzhou 325000, China \\ ${ }^{5}$ Cancer Center of Union Hospital, Tongji Medical College, Huazhong University of Science and Technology, Wuhan 430022, \\ China \\ ${ }^{6}$ Department of Endocrinology, The First Affiliated Hospital of Wenzhou Medical University, Wenzhou 325000, China \\ ${ }^{7}$ Department of Laboratory Medicine, The First Affiliated Hospital of Wenzhou Medical University, Wenzhou 325000, China \\ ${ }^{8}$ Global Medicines Development, AstraZeneca R \& D, Alderley Park, United Kingdom \\ ${ }^{9}$ Institute of Hepatology, Wenzhou Medical University, Wenzhou 325000, China \\ * Co-first authors, the author contributed equally to this work \\ Correspondence to: Ming-Hua Zheng, e-mail: zhengmh@wmu.edu.cn \\ Gui-Long Guo, e-mail: wzguoguilong@163.com
}

Keywords: colorectal cancer, platelet to lymphocyte ratio, overall survival, disease-free survival, prognostic biomarker

Received: December 04, 2015

Accepted: March 07, 2016

Published: March 24, 2016

\section{ABSTRACT}

Objectives: Recent studies suggest that an elevated preoperative platelet to lymphocyte ratio (PLR) may be considered a poor prognostic biomarker in patients with colorectal cancer (CRC). The aim of this study was to evaluate the prognostic impact of PLR in patients with CRC.

Methods: We enrolled 1314 patients who underwent surgery for CRC between 2005 and 2011. Preoperative PLR level was stratified into quintiles for Kaplan-Meier analysis and multivariable Cox proportional hazard regression models.

Results: Higher PLR quintiles were significantly associated with poorer overall survival $(P=0.002)$. Multivariate analysis showed that PLR was an independent risk factor for overall survival (OS) $(P=0.034)$. Patients in PLR quintile 5 had lower overall survival than in quintile 1 (hazard ratio $(H R)=1.701,95 \%$ confidence interval (CI): 1.267-2.282, $P<0.001$ ). Although patients in PLR quintile 5 had significantly lower disease-free survival (DFS) than in quintile 1 (HR = 1.522, 95\% CI: $1.114-$ 2.080, $P=0.008$ ), this association was not significant after multivariable adjustment $(P=0.075)$. In the subgroup analysis, PLR remained an independent factor in terms of advanced tumor stage (III, IV), male sex, carcinoembryonic antigen ( $\leq 5 \mathrm{ng} / \mathrm{ml}$ ), age ( $>65$ years) and body mass index $(\leq 25)(P<0.05$ for all measurements). The results remained unchanged when the PLR was analyzed as a dichotomous variable by applying different cut-off values of 150, 185, 220.

Conclusions: Elevated preoperative PLR was independently associated with an increased risk of mortality in patients with CRC. The utility of PLR may help to improve prognostic predictors. 


\section{INTRODUCTION}

Colorectal cancer (CRC) is the third malignant neoplasm in the world and more than 600,000 people die from this disease each year [1]. On the whole, the 5-year overall survival (OS) for patients with CRC ranged from $51 \%$ to $67 \%$, due to recurrence and metastasis of CRC $[2,3]$. Conventionally, some prognostic factors, such as tumor TNM stage, cell differentiation grade and vascular invasion have been widely utilized as predictors for the prognosis of CRC. However, the survival time varies widely even in patients with the same TNM stage and tumor differentiation grade. Therefore an urgent need remains to identify optimal biomarkers that can predict progression and prognosis of the disease as complementary tools to intervention.

Recently, a number of studies have provided to evidence in support of the concept that the host inflammatory response is associated with the development and progression of cancer [4-6] and moreover, with a poor outcome independent of the tumor stage $[7,8]$. Systemic inflammation can be assessed by means of peripheral blood markers such as serum white blood cells, neutrophils, lymphocyte and platelet and acute-phase proteins. Platelet to lymphocyte ratio (PLR) has been reported to be associated with poor prognosis in different tumor types, including CRC [9-14]. Nonetheless, results from several studies investigating the relationship between the PLR and the prognosis of patients with CRC remain inconsistent [15-18].

The primary objective of this study was to investigate the prognostic impact of the preoperative PLR on the survival in CRC patients and further validate the results of previous studies within a large cohort of CRC patients using different threshold values.

\section{RESULTS}

\section{Baseline characteristics}

Demographic and clinical characteristics of patients with CRC are listed in Table 1. There were 1314 eligible patients with available preoperative PLR levels. The mean age of patients was $66 \pm 12.6$ years, and the majority were male (59.7\%). 697 patients $(53.0 \%)$ were confirmed as presenting with rectal cancer. The majority of tumors exhibited moderate histological differentiation $(70.2 \%)$. At initial diagnosis, $16.0 \%$ of the CRC patients presented with stage I, followed by $38.3 \%$ with stage II, $37.7 \%$ with stage III, and $8.1 \%$ with stage IV.

The median preoperative PLR was 169.1. By applying receiver operating curve analysis, the optimal cut-off value for the PLR was 157.8 both for OS and for DFS. The cut off values for categorization of PLR into quintiles were 100,120,160 and 220. A higher PLR was significantly associated with lower values of
BMI at diagnosis $(P<0.01)$. Patients in PLR quintiles 5 were significantly associated with higher tumor stages, particularly stage IV disease. The tumors were also more likely to be associated with poor outcome predictors such as vascular invasion, total protein $(P<0.05$ for all measurements). There were no statistically significant differences in other clinic-pathological factors.

\section{The prognosis impact of the PLR on overall and disease-free survival}

The mean follow-up time was 59.6 months. KaplanMeier analysis of OS and DFS showed progressively worse OS with each PLR quintile ( $P=0.002$; Figure 1A). Patients with high DFS more likely linked with the low PLR, although the difference in DFS was not statistically significant $(P=0.078$; Figure 1B).

\section{Cox analyses of survival associated with PLR}

Unadjusted univariable Cox proportional hazard models were used to identify variables associated with OS and DFS and the results were presented in Table 2. The patients in highest quintile of PLR had $70 \%$ increase in hazard death and $52 \%$ increased hazard of having recurrence of disease compared with the first quintile (hazard ratio $(\mathrm{HR})=1.701 ; 95 \%$ confidence interval $(\mathrm{CI})$ $1.267-2.282, P<0.001$ and HR $=1.522 ; 95 \%$ CI 1.114 $2.080, P=0.008$, respectively). Gender, age, BMI, tumor TNM stage, tumor differentiation, the presence of vascular invasion, total protein and CEA were also significantly associated with the hazard of death in the univariate analysis $(P<0.05$ for all measurements). In the multivariate analysis, PLR remained significantly associated with OS (HR $=1.511 ; 95 \%$ CI 1.103-2.070, $P=0.010$ ). However, BMI and the presence of vascular invasion were not associated with OS. In the multivariate Cox analysis of DFS, gender, TNM stage, tumor differentiation and total protein were independent predictive risk factors for the prognosis of patients after adjustment for PLR, vascular invasion, and CEA $(P<0.05$ for all measurements, Table 2).

Based on the optimal cut-off values and those applied in previous studies $[14,15,17,18]$ we chose values of $150,185,220$ and 300 for the dichotomous analysis. Sensitivity analyses for the PLR with different cut of values returned qualitatively similar results (Table 3 ). In a adjusted multivariable analysis, CRC patients with the higher level of PLR were significantly associated with a higher risk of mortality compared with their counterparts with the lower PLR using the different cut-off values of 150,185 and $220(P=0.002, P=0.014, P=0.001$, respectively). However, by applying a cut-off of 300 , a high PLR was not significantly associated with the risk of mortality $(P=0.055)$. For DFS, using the cut-off values of 150 and 220, a high PLR was significantly associated 
Table 1: Characteristics of CRC patients treated by surgical resection according to PLR quintile

\begin{tabular}{|c|c|c|c|c|c|c|c|}
\hline \multirow[b]{2}{*}{ Characteristic } & \multirow[b]{2}{*}{$\begin{array}{l}\text { All patients } \\
\quad N(\%)\end{array}$} & \multicolumn{5}{|c|}{ PLR quintiles } & \multirow[b]{2}{*}{$P$ value } \\
\hline & & $\begin{array}{c}\text { Quintile 1 } \\
\text { PLR } \leq \mathbf{1 0 0} \\
N=\mathbf{2 9 7}\end{array}$ & $\begin{array}{c}\text { Quintile 2 } \\
100<\text { PLR } \leq 120 \\
N=\mathbf{2 0 5}\end{array}$ & $\begin{array}{c}\text { Quintile } 3 \\
120<\text { PLR } \leq 160 \\
N=296\end{array}$ & $\begin{array}{c}\text { Quintile } 4 \\
160<\text { PLR } \leq 220 \\
N=256\end{array}$ & $\begin{array}{c}\text { Quintile 5 } \\
\text { PLR > 220 } \\
N=\mathbf{2 6 0}\end{array}$ & \\
\hline Median PLR & 169.1 & 79.4 & 110.1 & 139.3 & 186.5 & 334.9 & \\
\hline Age (mean \pm SD) & $66.0 \pm 12.6$ & $66.7 \pm 12.0$ & $66.2 \pm 11.5$ & $66.4 \pm 12.5$ & $65.3 \pm 13.2$ & $66.5 \pm 13.7$ & 0.655 \\
\hline \multicolumn{7}{|l|}{ Gender } & \multirow[t]{3}{*}{0.959} \\
\hline Male, $n(\%)$ & $785(59.7 \%)$ & $178(59.9 \%)$ & $124(60.5 \%)$ & $181(61.1 \%)$ & $149(58.2 \%)$ & $153(58.8 \%)$ & \\
\hline Female, $n(\%)$ & $529(40.3 \%)$ & $119(40.1 \%)$ & $81(39.5 \%)$ & $115(38.9 \%)$ & $107(41.8 \%)$ & $107(41.2 \%)$ & \\
\hline BMI $(\mathrm{kg} / \mathrm{m} 2)$ & $21.9 \pm 3.4$ & $22.2 \pm 3.1$ & $22.1 \pm 3.4$ & $22.1 \pm 3.4$ & $21.8 \pm 3.6$ & $21.1 \pm 3.1$ & $<0.001$ \\
\hline Obesity, $n(\%)$ & $185(14.1 \%)$ & $46(15.5 \%)$ & $37(18.0 \%)$ & $44(14.9 \%)$ & $33(12.9 \%)$ & $25(9.6 \%)$ & 0.096 \\
\hline Hypertension, $n(\%)$ & $369(28.1 \%)$ & $87(29.3 \%)$ & $54(26.3 \%)$ & $94(31.8 \%)$ & $73(28.5 \%)$ & $61(23.5 \%)$ & 0.260 \\
\hline $\mathrm{DM}, n(\%)$ & $128(9.7 \%)$ & $31(10.4 \%)$ & $16(7.8 \%)$ & $30(10.1 \%)$ & $24(9.4 \%)$ & $27(10.4 \%)$ & 0.870 \\
\hline \multicolumn{7}{|l|}{ TNM Staging } & \multirow[t]{5}{*}{0.008} \\
\hline Stage I, $n(\%)$ & $210(16.0 \%)$ & $59(19.9 \%)$ & $42(20.5 \%)$ & $42(14.2 \%)$ & $39(15.2 \%)$ & $28(10.8 \%)$ & \\
\hline Stage II, $n(\%)$ & $503(38.3 \%)$ & $115(38.7 \%)$ & $63(30.7 \%)$ & $124(41.9 \%)$ & $93(36.3 \%)$ & $108(41.5 \%)$ & \\
\hline Stage III, $n(\%)$ & $495(37.7 \%)$ & $104(35.0 \%)$ & $90(43.9 \%)$ & $105(35.5 \%)$ & $102(39.8 \%)$ & $94(36.2 \%)$ & \\
\hline Stage IV, $n(\%)$ & $106(8.1 \%)$ & $19(6.4 \%)$ & $10(4.9 \%)$ & $25(8.4 \%)$ & $22(8.6 \%)$ & $30(11.5 \%)$ & \\
\hline $\begin{array}{l}\text { Histological } \\
\text { differentiation }\end{array}$ & & & & & & & \multirow[t]{4}{*}{0.447} \\
\hline Well, $n(\%)$ & $43(3.3 \%)$ & $13(4.4 \%)$ & $6(2.9 \%)$ & $11(3.7 \%)$ & $5(2.0 \%)$ & $8(3.1 \%)$ & \\
\hline Moderately, $n(\%)$ & $923(70.2 \%)$ & $218(73.4 \%)$ & $140(68.3 \%)$ & $208(70.3 \%)$ & $184(71.9 \%)$ & $173(66.5 \%)$ & \\
\hline Poorly, $n(\%)$ & $348(26.5 \%)$ & $66(22.2 \%)$ & $59(28.8 \%)$ & $77(26.0 \%)$ & $67(26.2 \%)$ & $79(30.4 \%)$ & \\
\hline Vascular invasion, $n(\%)$ & $185(14.1 \%)$ & $29(9.8 \%)$ & $23(11.2 \%)$ & $46(15.5 \%)$ & $48(18.8 \%)$ & $39(15.0 \%)$ & 0.024 \\
\hline \multicolumn{7}{|l|}{ Location } & \multirow[t]{4}{*}{$<0.001$} \\
\hline Right side, $n(\%)$ & $208(15.8 \%)$ & $32(10.8 \%)$ & $18(8.8 \%)$ & $44(14.9 \%)$ & $44(17.2 \%)$ & $70(26.9 \%)$ & \\
\hline Sigmoid, $n(\%)$ & $232(17.7 \%)$ & $48(16.2 \%)$ & $41(20.0 \%)$ & $56(18.9 \%)$ & $45(17.6 \%)$ & $42(16.2 \%)$ & \\
\hline Rectal, $n(\%)$ & $697(53.0 \%)$ & $183(61.6 \%)$ & $129(62.9 \%)$ & $164(55.4 \%)$ & $125(48.8 \%)$ & $96(36.9 \%)$ & \\
\hline $\mathrm{CEA}(\mathrm{ng} / \mathrm{ml})$ & $29.8 \pm 146.7$ & $24.1 \pm 136.7$ & $35.6 \pm 170.8$ & $30.9 \pm 111.7$ & $28.4 \pm 173.4$ & $31.8 \pm 144.6$ & 0.938 \\
\hline Creatinine $(\mu \mathrm{mol} / \mathrm{L})$ & $68.0 \pm 33.3$ & $68.2 \pm 31.2$ & $68.9 \pm 28.8$ & $70.9 \pm 40.0$ & $66.3 \pm 23.1$ & $65.3 \pm 38.5$ & 0.327 \\
\hline Total protein $(\mathrm{g} / \mathrm{L})$ & $67.8 \pm 7.2$ & $69.7 \pm 6.0$ & $68.8 \pm 6.3$ & $68.0 \pm 7.8$ & $67.6 \pm 6.5$ & $65.2 \pm 8.2$ & $<0.001$ \\
\hline
\end{tabular}

Abbreviations: $\mathrm{CRC}=$ colorectal cancer, $\mathrm{PLR}=$ platelet lymphocyte ratio, $\mathrm{BMI}=$ body mass index, $\mathrm{DM}=$ diabetes $\mathrm{mellitus}$, $\mathrm{CEA}=$ carcinoembryonic antigen

with the risk of disease recurrence $(P=0.033, P=0.024$, respectively) (Table 3 ). Sensitivity analyses using this different set of the PLR quintiles (cut-off values 150, 185, $220,300)$ did not change the main results (Supplementary Figure 1).

\section{Subgroup analyses associated with PLR}

We used Kaplan-Meier methodology to examine the impact of PLR on OS in patients stratified by age, BMI, tumor differentiation, gender, tumor stage and CEA, PLR quintiles were significantly associated with poor survival in older ( $>65$ years) (Figure 2B) male (Figure 3A) patients with advanced tumor TNM stage (III and IV) (Figure 4B), normal range of CEA $(\leq 5 \mathrm{ng} / \mathrm{ml})$ (Figure $5 \mathrm{~A}$ ) and BMI $(\leq 25)$ (Figure 6A) $(P<0.01$ for all measurements).
When stratified by tumor differentiation, PLR was closely associated with poor survival in patients with well and moderate differentiation, although not significantly $(P=0.058)$ (Figure 7A).

When compared with the quintile representing the lowest value of PLR, CRC patients in the highest quintile of the PLR were also found to have substantially lower survival rates in several aspects, such as male $(\mathrm{HR}=2.116,95 \% \mathrm{CI} 1.461-3.064), \mathrm{BMI}(\leq 25)(\mathrm{HR}=1.785$, $95 \%$ CI $1.302-2.445)$, tumor stage (III and IV) $(\mathrm{HR}=1.657$, $95 \%$ CI $1.166-2.354$ ), tumor differentiation (well and moderately) $(\mathrm{HR}=1.511,95 \%$ CI $1.060-2.152)$, CEA $(\leq 5 \mathrm{ng} / \mathrm{ml})(\mathrm{HR}=2.403,95 \%$ CI $1.533-3.768)$, irrespective of age $(\geq 65)(\mathrm{HR}=1.776,95 \%$ CI $1.213-2.601)$ or young age $(<65)(\mathrm{HR}=1.622,95 \%$ CI $1.022-2.574)$ (Table 4). 
Table 2: Cox proportional hazards regression models of risk factors associated with overall and disease-free survival among CRC patients

\begin{tabular}{|c|c|c|c|c|c|c|c|c|c|c|c|c|}
\hline & \multicolumn{6}{|c|}{ Overall Survival } & \multicolumn{6}{|c|}{ Disease-free Survival } \\
\hline & \multicolumn{3}{|c|}{ Univariable } & \multicolumn{3}{|c|}{ Multivariable } & \multicolumn{3}{|c|}{ Univariable } & \multicolumn{3}{|c|}{ Multivariable } \\
\hline & HR & $95 \%$ CI & $P$ & HR & $95 \%$ CI & $P$ & HR & $95 \%$ CI & $P$ & HR & $95 \%$ CI & $P$ \\
\hline $\begin{array}{l}\text { Gender } \\
\text { (male vs female) }\end{array}$ & 1.389 & $1.133-1.704$ & 0.002 & 1.331 & $1.078-1.645$ & 0.008 & 1.372 & $1.106-1.701$ & 0.004 & 1.307 & $1.046-1.632$ & 0.018 \\
\hline Age & 1.011 & $1.003-1.019$ & 0.009 & 1.011 & 1.003-1.020 & 0.007 & 1.007 & $0.998-1.015$ & 0.111 & & & \\
\hline BMI $(\mathrm{kg} / \mathrm{m} 2)$ & 0.968 & $0.939-0.998$ & 0.035 & & & & 0.984 & $0.954-1.016$ & 0.328 & & & \\
\hline PLR(continuous) & 1.001 & $1.000-1.001$ & 0.040 & 1.001 & $1.000-1.002$ & 0.034 & 1.001 & $1.000-1.001$ & 0.100 & 1.001 & $1.000-1.002$ & 0.078 \\
\hline Quintile 1 & 1.000 & - & - & 1.000 & - & - & 1.000 & - & - & 1.000 & - & - \\
\hline Quintile 2 & 1.050 & $0.746-1.479$ & 0.778 & 0.823 & $0.578-1.173$ & 0.281 & 1.110 & $0.784-1.574$ & 0.557 & 0.906 & $0.629-1.303$ & 0.593 \\
\hline Quintile 3 & 1.146 & $0.844-1.554$ & 0.383 & 1.038 & $0.840-1.605$ & 0.816 & 1.108 & $0.805-1.525$ & 0.528 & 1.047 & $0.752-1.459$ & 0.785 \\
\hline Quintile 4 & 1.318 & $0.966-1.797$ & 0.081 & 1.161 & $0.840-1.605$ & 0.365 & 1.246 & $0.899-1.728$ & 0.186 & 1.126 & $0.800-1.584$ & 0.497 \\
\hline Quintile 5 & 1.701 & $1.267-2.282$ & $<0.001$ & 1.511 & 1.103-2.070 & 0.010 & 1.522 & $1.114-2.080$ & 0.008 & 1.356 & $0.970-1.896$ & 0.075 \\
\hline TNM Staging & 0.220 & $0.176-0.275$ & $<0.001$ & 0.243 & $0.192-0.307$ & $<0.001$ & 0.322 & $0.260-0.398$ & $<0.001$ & 0.352 & $0.280-0.442$ & $<0.001$ \\
\hline Stage I & 1.000 & - & - & & & & 1.000 & - & - & & & \\
\hline Stage II & 1.009 & $0.6640-1.533$ & 0.967 & & & & 1.022 & $0.805-1.484$ & 0.907 & & & \\
\hline Stage III & 3.438 & $2.356-5.016$ & $<0.001$ & & & & 3.119 & $2.220-4.381$ & $<0.001$ & & & \\
\hline Stage IV & 17.431 & 11.575-26.248 & $<0.001$ & & & & 11.790 & 4.199-33.102 & $<0.001$ & & & \\
\hline Differentiation & 0.580 & $0.474-0.711$ & $<0.001$ & 0.752 & $0.607-0.932$ & 0.009 & 0.604 & $0.486-0.750$ & $<0.001$ & 0.735 & $0.584-0.926$ & 0.009 \\
\hline $\begin{array}{l}\text { Vascular } \\
\text { invasion }\end{array}$ & 0.528 & $0.416-0.669$ & $<0.001$ & & & & 0.608 & $0.465-0.795$ & $<0.001$ & & & \\
\hline Total protein & 0.980 & $0.967-0.993$ & 0.002 & 0.984 & $0.970-0.998$ & 0.022 & 0.978 & $0.965-0.992$ & 0.002 & 0.978 & $0.964-0.993$ & 0.003 \\
\hline CEA & 1.000 & $1.000-1.001$ & $<0.001$ & 1.001 & 1.001-1.001 & $<0.001$ & 1.000 & $1.000-1.001$ & $<0.001$ & 1.000 & $1.000-1.001$ & 0.309 \\
\hline Creatinine & 1.000 & $0.997-1.003$ & 0.958 & & & & 1.001 & $0.998-1.004$ & 0.536 & & & \\
\hline $\mathrm{DM}$ & 0.980 & $0.707-1.358$ & 0.905 & & & & 0.948 & $0.672-1.338$ & 0.763 & & & \\
\hline Hypertension & 0.873 & $0.707-1.078$ & 0.206 & & & & 0.837 & $0.670-1.046$ & 0.117 & & & \\
\hline Obesity & 0.938 & $0.717-1.227$ & 0.641 & & & & 1.159 & $0.876-1.535$ & 0.301 & & & \\
\hline
\end{tabular}

Abbreviations: $\mathrm{CRC}=$ colorectal cancer, $\mathrm{PLR}=$ platelet lymphocyte ratio, $\mathrm{BMI}=$ body mass index, $\mathrm{CEA}=$ carcinoembryonic antigen, $\mathrm{DM}=$ diabetes mellitus, $\mathrm{HR}=$ hazard ratio, $\mathrm{CI}=$ confidence interval.

Table 3: Association between PLR and mortality in CRC patients applying different cutoff values

\begin{tabular}{|c|c|c|c|c|c|c|c|c|c|c|}
\hline \multirow{3}{*}{ PLR } & \multicolumn{5}{|c|}{ Overall Survival } & \multicolumn{5}{|c|}{ Disease-free Survival } \\
\hline & \multirow{2}{*}{ Total } & \multicolumn{2}{|c|}{ Univariable } & \multicolumn{2}{|c|}{ Multivariable* } & \multirow{2}{*}{ Total } & \multicolumn{2}{|c|}{ Univariable } & \multicolumn{2}{|c|}{ Multivariable* } \\
\hline & & HR 95\% CI & $\boldsymbol{P}$ & HR 95\% CI & $\boldsymbol{P}$ & & HR 95\% CI & $\boldsymbol{P}$ & HR 95\% CI & $\boldsymbol{P}$ \\
\hline$\leq 150$ & 747 & 1.000 & \multirow[b]{2}{*}{0.001} & 1.000 & \multirow[b]{2}{*}{0.002} & 700 & 1.000 & \multirow[b]{2}{*}{0.024} & 1.000 & \multirow[b]{2}{*}{0.033} \\
\hline$>150$ & 567 & $\begin{array}{c}1.387 \\
(1.143-1.682)\end{array}$ & & $\begin{array}{c}1.378 \\
(1.123-1.691)\end{array}$ & & 513 & $\begin{array}{c}1.267 \\
(1.032-1.556)\end{array}$ & & $\begin{array}{c}1.267 \\
(1.020-1.575)\end{array}$ & \\
\hline$\leq 185$ & 932 & 1.000 & \multirow[b]{2}{*}{0.002} & 1.000 & \multirow[b]{2}{*}{0.014} & 816 & 1.000 & \multirow[b]{2}{*}{0.022} & 1.000 & \multirow[b]{2}{*}{0.069} \\
\hline$>185$ & 382 & $\begin{array}{c}1.380 \\
(1.126-1.691)\end{array}$ & & $\begin{array}{c}1.314 \\
(1.056-1.634)\end{array}$ & & 316 & $\begin{array}{c}1.289 \\
(1.037-1.603)\end{array}$ & & $\begin{array}{c}1.241 \\
(0.983-1.567)\end{array}$ & \\
\hline$\leq 220$ & 1054 & 1.000 & \multirow[b]{2}{*}{0.001} & 1.000 & \multirow[b]{2}{*}{0.001} & 981 & 1.000 & \multirow[b]{2}{*}{0.010} & 1.000 & \multirow[b]{2}{*}{0.024} \\
\hline$>220$ & 260 & $\begin{array}{c}1.511 \\
(1.211-1.887)\end{array}$ & & $\begin{array}{c}1.492 \\
(1.174-1.896)\end{array}$ & & 232 & $\begin{array}{c}1.372 \\
(1.079-1.745)\end{array}$ & & $\begin{array}{c}1.346 \\
(1.040-1.742)\end{array}$ & \\
\hline$\leq 300$ & 1207 & 1.000 & \multirow[b]{2}{*}{0.179} & 1.000 & \multirow[b]{2}{*}{0.055} & 1135 & 1.000 & \multirow[b]{2}{*}{0.397} & 1.000 & \multirow[b]{2}{*}{0.155} \\
\hline$>300$ & 107 & $\begin{array}{c}1.254 \\
(0.902-1.744)\end{array}$ & & $\begin{array}{c}1.408 \\
(0.993-1.995)\end{array}$ & & 93 & $\begin{array}{c}1.167 \\
(0.816-1.669)\end{array}$ & & $\begin{array}{c}1.312 \\
(0.903-1.906)\end{array}$ & \\
\hline
\end{tabular}

Abbreviations: $\mathrm{CRC}=$ colorectal cancer, $\mathrm{PLR}=$ platelet lymphocyte ratio, $\mathrm{HR}=$ hazard ratio, $\mathrm{CI}=$ confidence interval.

*Hazard ratios for PLR were derived using Cox regression adjusted for age at diagnosis, gender, TNM stages, tumor differentiation, the presence of vascular invasion, total protein, CEA. 
Table 4: Cox proportional hazard regression analysis of OS in CRC patients stratified by clinic-pathological variables according to PLR quintiles

\begin{tabular}{|c|c|c|c|c|c|c|c|}
\hline \multirow{2}{*}{ Characteristic } & \multirow{2}{*}{ Total } & \multicolumn{5}{|c|}{ PLR quintiles } & \multirow{2}{*}{$\begin{array}{l}P \text { value } \\
\text { trend }^{a}\end{array}$} \\
\hline & & Quintile 1 & Quintile 2 & Quintile 3 & Quintile 4 & Quintile 5 & \\
\hline \multicolumn{8}{|l|}{ Age (years) } \\
\hline$>65$ & 697 & 1.00 & $1.112(0.711-1.740)$ & $1.046(0.698-1.567)$ & $1.476(0.991-2.199)$ & $1.776(1.213-2.601)^{\mathrm{b}}$ & 0.011 \\
\hline$\leq 65$ & 617 & 1.00 & $1.001(0.588-1.706)$ & $1.294(0.812-2.063)$ & $1.145(0.699-1.877)$ & $1.622(1.022-2.574)^{\mathrm{b}}$ & 0.218 \\
\hline \multicolumn{8}{|l|}{ Gender } \\
\hline Male & 785 & 1.00 & $1.260(0.825-1.926)$ & $1.255(0.852-1.848)$ & $1.476(0.994-2.191)$ & $2.116(1.461-3.064)^{\mathrm{b}}$ & 0.001 \\
\hline Female & 529 & 1.00 & $0.756(0.418-1.366)$ & $0.942(0.568-1.563)$ & $1.109(0.671-1.833)$ & $1.182(0.724-1.930)$ & 0.619 \\
\hline \multicolumn{8}{|l|}{ BMI (kg/m2) } \\
\hline$>25$ & 185 & 1.00 & $0.934(0.409-2.132)$ & $1.377(0.662-2.863)$ & $1.622(0.761-3.453)$ & $1.074(0.428-2.691)$ & 0.610 \\
\hline$\leq 25$ & 1129 & 1.00 & $1.076(0.738-1.568)$ & $1.108(0.792-1.550)$ & $1.272(0.905-1.788)$ & $1.785(1.302-2.445)^{\mathrm{b}}$ & 0.001 \\
\hline \multicolumn{8}{|l|}{ TNM Staging } \\
\hline Stage I, II & 713 & 1.00 & $0.910(0.470-1.759)$ & $0.870(0.484-1.563)$ & $0.981(0.532-1.807)$ & $1.560(0.908-2.679)$ & 0.229 \\
\hline Stage III, IV & 601 & 1.00 & $0.982(0.657-1.468)$ & $1.238(0.864-1.773)$ & $1.329(0.925-1.911)$ & $1.657(1.166-2.354)^{\mathrm{b}}$ & 0.005 \\
\hline \multicolumn{8}{|l|}{ Differentiation } \\
\hline Well and Moderately & 966 & 1.00 & $0.913(0.600-1.388)$ & $0.985(0.682-1.422)$ & $1.129(0.777-1.642)$ & $1.511(1.060-2.152)^{\mathrm{b}}$ & 0.062 \\
\hline Poorly, & 348 & 1.00 & $0.738(0.433-1.258)$ & $0.807(0.494-1.318)$ & $0.582(0.334-1.013)$ & $0.946(0.585-1.530)$ & 0.320 \\
\hline \multicolumn{8}{|l|}{ CEA (ng/ml) } \\
\hline$>5$ & 484 & 1.000 & $0.930(0.580-1.489)$ & $0.917(0.607-1.384)$ & $1.363(0.901-2.062)$ & $1.210(0.798-1.834)$ & 0.255 \\
\hline$\leq 5$ & 751 & 1.000 & $1.145(0.673-1.947)$ & $1.347(0.834-2.173)$ & $1.193(0.719-1.979)$ & $2.403(1.533-3.768)^{\mathrm{b}}$ & $<0.001$ \\
\hline
\end{tabular}

Abbreviations: PLR = platelet lymphocyte ratio, $\mathrm{OS}=$ overall survival, $\mathrm{BMI}=$ body mass index, $\mathrm{CEA}=$ carcinoembryonic antigen, $\mathrm{CI}=$ confidence interval

${ }^{a} \mathrm{P}$ for trend is computed by entering the quintiles as discontinuous terms in the Cox model

${ }^{\mathrm{b} S}$ Statistically significant $(P<0.05)$
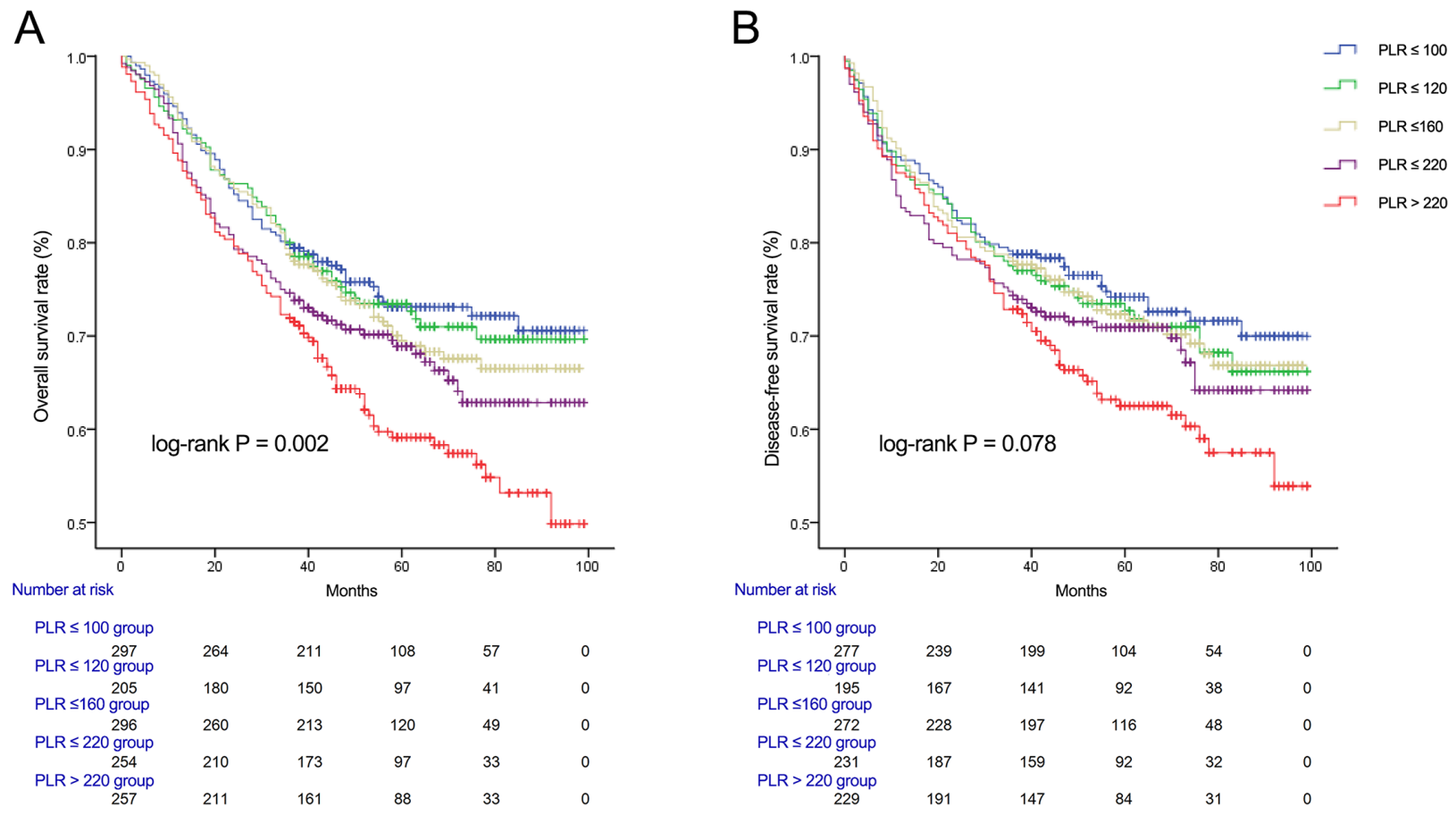

\begin{tabular}{|c|c|c|c|c|c|}
\hline$P L R \leq 100$ group & & & & & \\
\hline $\mathrm{PLR} \leq 120^{277}$ group & 239 & 199 & 104 & 54 & 0 \\
\hline $\begin{array}{c}195 \\
\mathrm{PLR} \leq 160 \text { group }\end{array}$ & 167 & 141 & 92 & 38 & 0 \\
\hline $\begin{array}{c}272 \\
P L R \leq 220 \text { group }\end{array}$ & 228 & 197 & 116 & 48 & 0 \\
\hline $\begin{array}{c}231 \\
\mathrm{PIR}>220 \text { roup }\end{array}$ & 187 & 159 & 92 & 32 & 0 \\
\hline 229 & 191 & 147 & 84 & 31 & 0 \\
\hline
\end{tabular}

Figure 1: Kaplan-Meier survival curves showing overall survival (A) and disease-free survival (B) stratified by quintiles of PLR in colorectal cancer patients. 

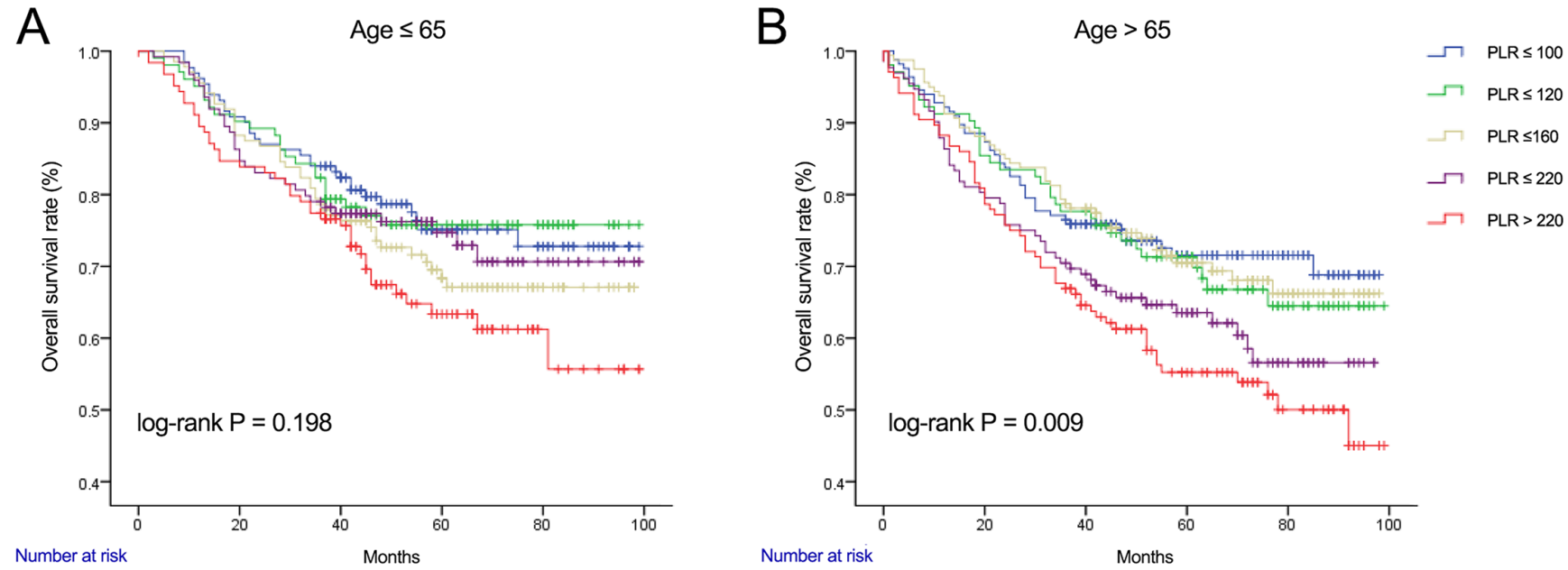

\begin{tabular}{|c|c|c|c|c|c|}
\hline Number at risk & & & & & \\
\hline$P L R \leq 100$ group & & & & & \\
\hline$P L R \leq 120$ group & 119 & 98 & 48 & 25 & 0 \\
\hline $\begin{array}{c}102 \\
P L R \leq 160 \text { group }\end{array}$ & 92 & 71 & 44 & 20 & 0 \\
\hline $\begin{array}{l}136 \\
P L R \leq 220 \text { group }\end{array}$ & 120 & 95 & 54 & 20 & 0 \\
\hline PLR $>220$ group & 105 & 85 & 46 & 15 & 0 \\
\hline 123 & 104 & 82 & 40 & 11 & 0 \\
\hline
\end{tabular}

\begin{tabular}{|c|c|c|c|c|c|}
\hline$P L R \leq 100$ group & & & & & \\
\hline$P L R \leq 120^{166}$ group & 145 & 113 & 60 & 32 & 0 \\
\hline $\begin{array}{c}103 \\
P L R \leq 160 \text { group }\end{array}$ & 88 & 79 & 53 & 21 & 0 \\
\hline $\begin{array}{c}160 \\
P L R \leq 220 \text { group }\end{array}$ & 140 & 118 & 66 & 29 & 0 \\
\hline $\begin{array}{c}130 \\
\text { PLR }>220 \text { group }\end{array}$ & 105 & 88 & 51 & 18 & 0 \\
\hline 134 & 107 & 79 & 48 & 22 & 0 \\
\hline
\end{tabular}

Figure 2: Overall survival of CRC patients stratified by quintiles of PLR according to (A) young age $(\leq 65)$ and (B) old age ( $>65)$.
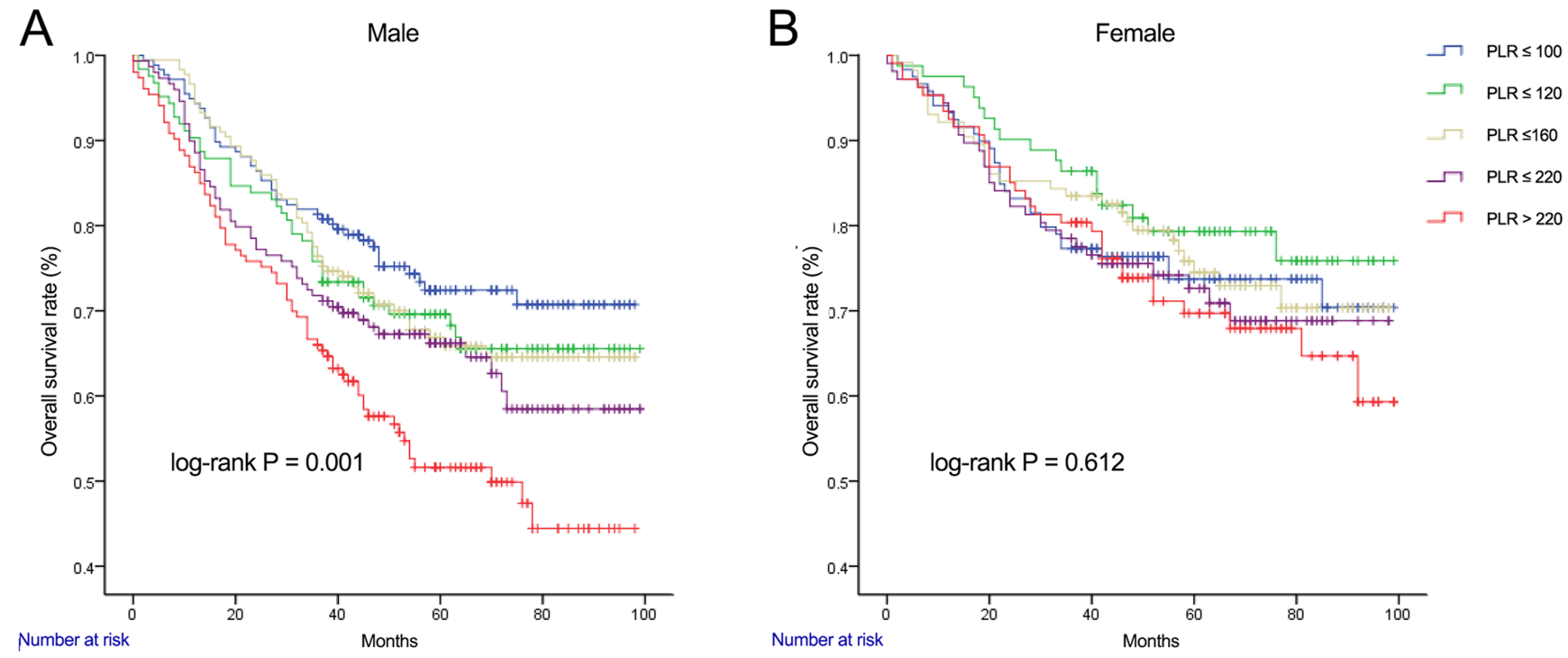

\begin{tabular}{|c|c|c|c|c|c|}
\hline \multicolumn{6}{|l|}{$P L R \leq 100$ group } \\
\hline$P L R \leq 120$ group & 157 & 126 & 59 & 30 & 0 \\
\hline PLR $\leq 160$ group & 105 & 85 & 55 & 24 & 0 \\
\hline $\begin{array}{c}178 \\
\mathrm{PLR} \leq 220 \text { group }\end{array}$ & 159 & 122 & 66 & 28 & 0 \\
\hline $\begin{array}{c}148 \\
\text { PLR }>220 \text { group }\end{array}$ & 119 & 97 & 52 & 19 & 0 \\
\hline 150 & 118 & 86 & 41 & 12 & 0 \\
\hline
\end{tabular}

$\begin{array}{cccccc}\text { PLR } \leq 100 \text { group } & & & & & \\ \begin{array}{c}119 \\ \text { PLR } \leq 120 \text { group }\end{array} & 106 & 84 & 48 & 26 & 0 \\ \begin{array}{c}81 \\ \text { PLR } \leq 160 \text { group }\end{array} & 75 & 65 & 42 & 17 & 0 \\ \begin{array}{c}115 \\ \text { PLR } \leq 220 \text { group } \\ 106\end{array} & 99 & 90 & 53 & 20 & 0 \\ \text { PLR }>220 \text { group } & 91 & 76 & 45 & 14 & 0 \\ 107 & 93 & 75 & 47 & 21 & 0\end{array}$

Figure 3: Overall survival of CRC patients stratified by quintiles of PLR according to male (A) and female (B). 

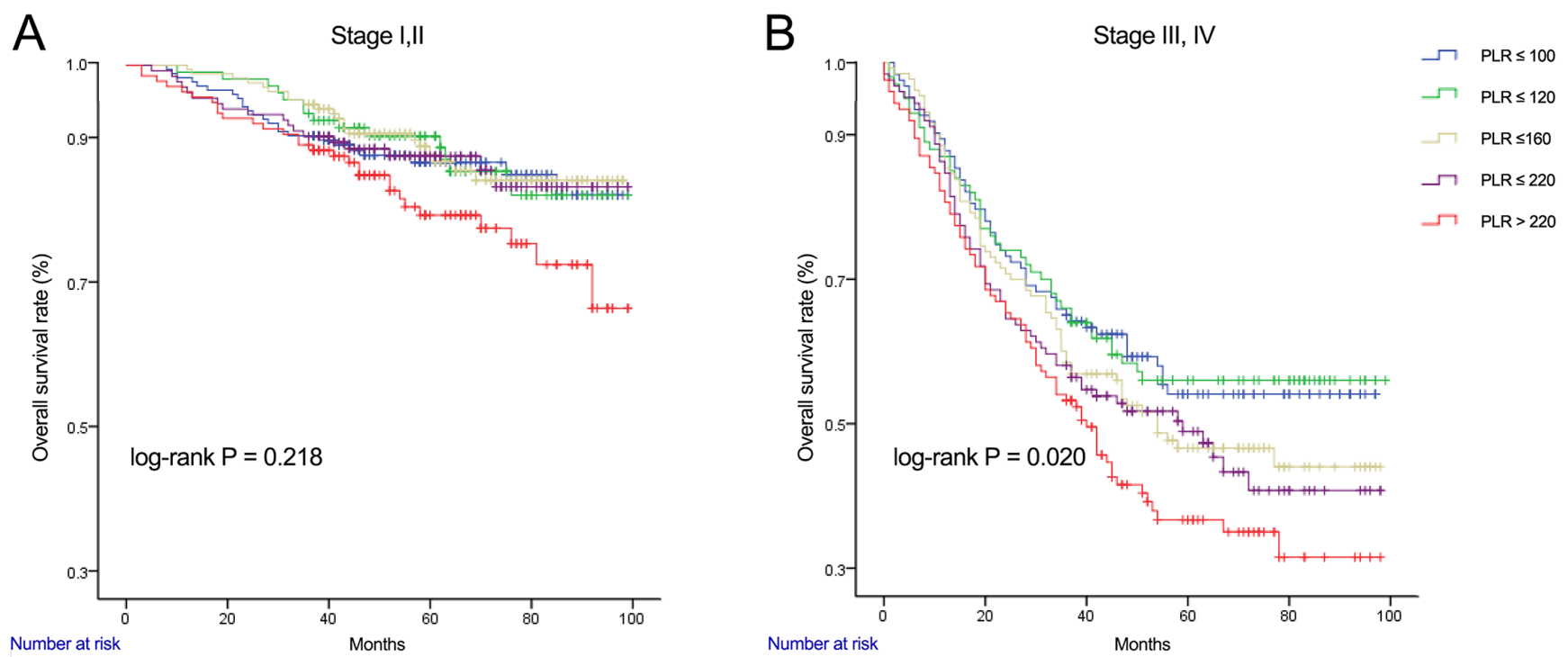

\begin{tabular}{|c|c|c|c|c|c|}
\hline $\begin{array}{c}P L R \leq 100 \text { group } \\
174\end{array}$ & 168 & 140 & 72 & 37 & 0 \\
\hline$P L R \leq 120$ group & & & & & \\
\hline $\begin{array}{c}105 \\
\mathrm{PLR} \leq 160 \text { group }\end{array}$ & 103 & 91 & 62 & 19 & 0 \\
\hline $\begin{array}{c}166 \\
P L R \leq 220 \text { group }\end{array}$ & 164 & 142 & 80 & 37 & 0 \\
\hline $\begin{array}{c}132 \\
\text { PLR }>220 \text { group }\end{array}$ & 124 & 110 & 64 & 24 & 0 \\
\hline 136 & 126 & 108 & 62 & 26 & 0 \\
\hline
\end{tabular}

\begin{tabular}{|c|c|c|c|c|c|}
\hline$P L R \leq 100$ group & & & & & \\
\hline$P L R \leq 120$ group & 96 & 71 & 36 & 20 & 0 \\
\hline $\begin{array}{c}100 \\
P L R \leq 160 \text { group }\end{array}$ & 77 & 59 & 35 & 22 & 0 \\
\hline $\begin{array}{c}130 \\
P L R \leq 220 \text { group }\end{array}$ & 96 & 71 & 40 & 12 & 0 \\
\hline $\begin{array}{c}122 \\
P L R>220 \text { group }\end{array}$ & 86 & 63 & 33 & 9 & 0 \\
\hline 121 & 85 & 53 & 26 & 7 & 0 \\
\hline
\end{tabular}

Figure 4: Overall survival of CRC patients stratified by quintiles of PLR according to tumor stage I,II (A) and tumor stage III,IV (B).

A

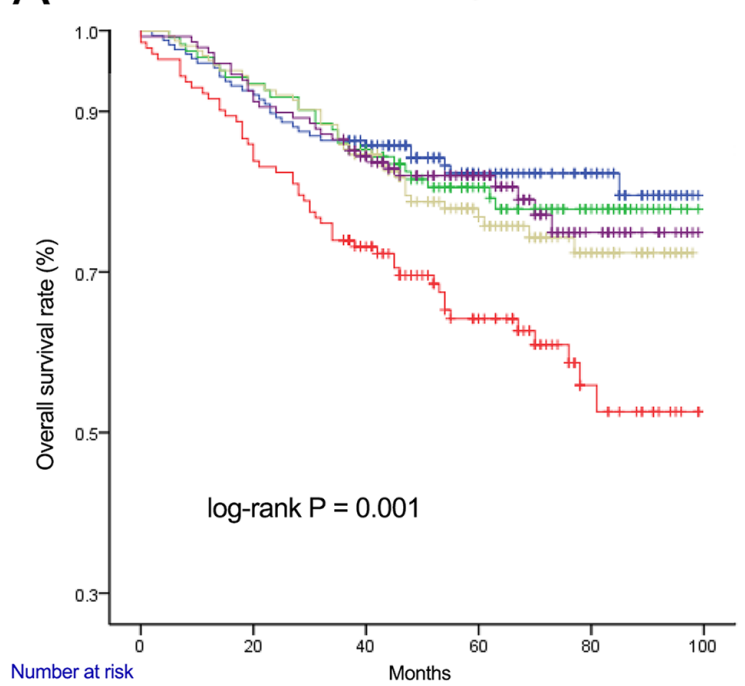

Number at risk

\begin{tabular}{|c|c|c|c|c|c|}
\hline$P L R \leq 100$ group & & & & & \\
\hline$P L R \leq 120$ group & 162 & 136 & 67 & 37 & 0 \\
\hline PLR $\leq 160$ group & 114 & 98 & 62 & 27 & 0 \\
\hline $\begin{array}{c}163 \\
P L R \leq 220 \text { group }\end{array}$ & 152 & 127 & 68 & 31 & $n$ \\
\hline $\begin{array}{c}147 \\
P L R>220 \text { group }\end{array}$ & 135 & 115 & 68 & 22 & u \\
\hline 140 & 119 & 88 & 53 & 17 & 0 \\
\hline
\end{tabular}

B

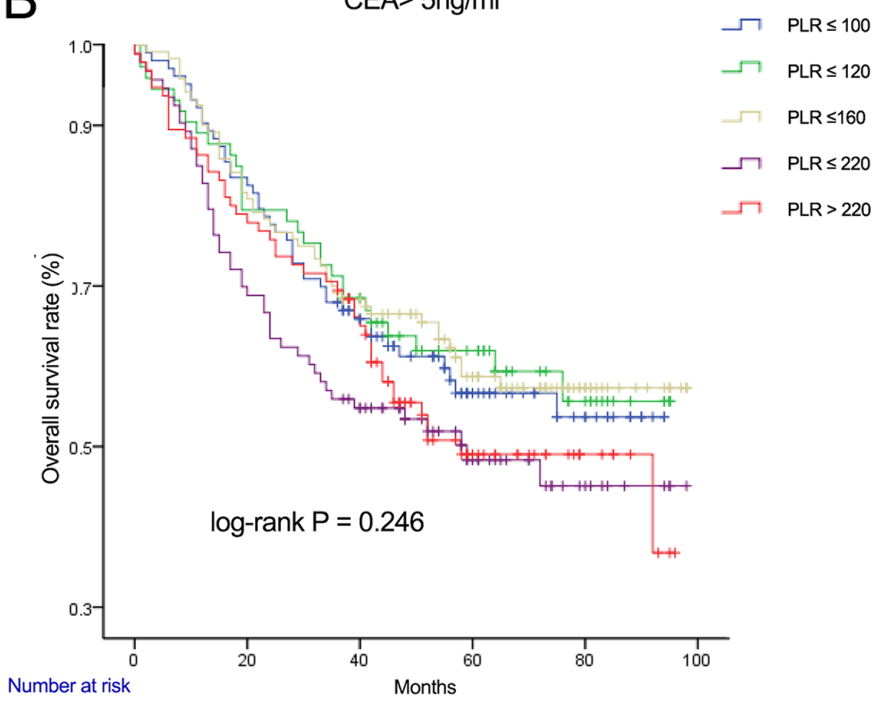

Figure 5: Overall survival of CRC patients stratified by quintiles of PLR according to CEA $\leq 5 \mathrm{ng} / \mathrm{ml}$ (A) and CEA $>5 \mathrm{ng} / \mathrm{ml}(\mathrm{B})$. 
A

$\mathrm{BMl} \leq 25$

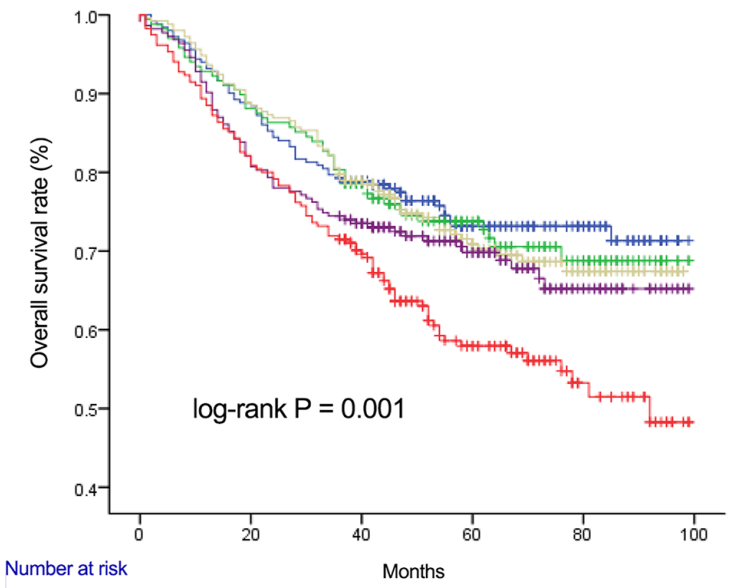

\begin{tabular}{|c|c|c|c|c|c|}
\hline$P L R \leq 100$ group & & & & & \\
\hline $\begin{array}{c}251 \\
P L R \leq 120 \text { group }\end{array}$ & 222 & 180 & 90 & 50 & 0 \\
\hline $\begin{array}{c}168 \\
\text { PLR } \leq 160 \text { group }\end{array}$ & 148 & 124 & 76 & 34 & 0 \\
\hline $\begin{array}{c}252 \\
P L R \leq 220 \text { group }\end{array}$ & 223 & 185 & 105 & 45 & 0 \\
\hline 221 & 180 & 153 & 87 & 32 & 0 \\
\hline$P L R>\underset{233}{220}$ group & 190 & 147 & 79 & 30 & 0 \\
\hline
\end{tabular}

B

B $\quad B M I>25$

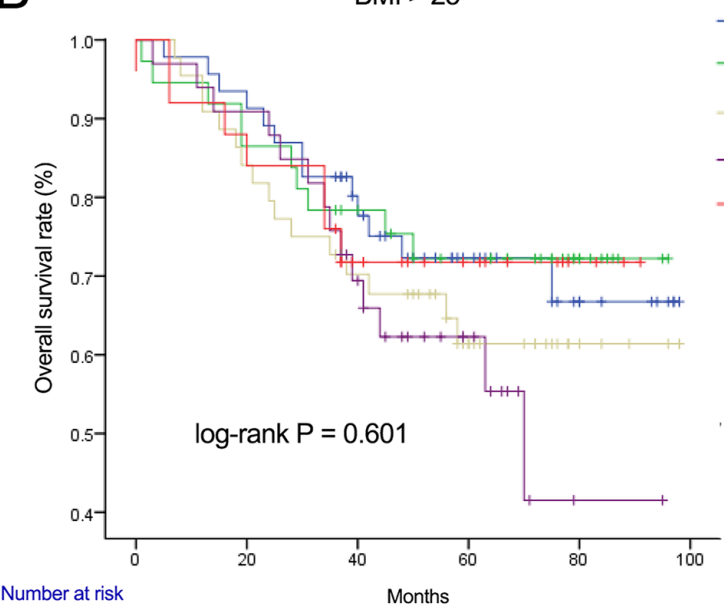

Number at risk

Months

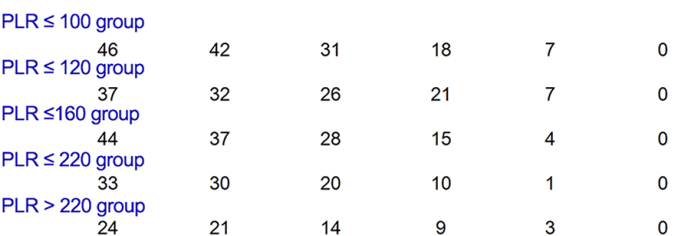

Figure 6: Overall survival of CRC patients stratified by quintiles of PLR according to $B M I \leq 25$ (A) and $B M I>25$ (B).
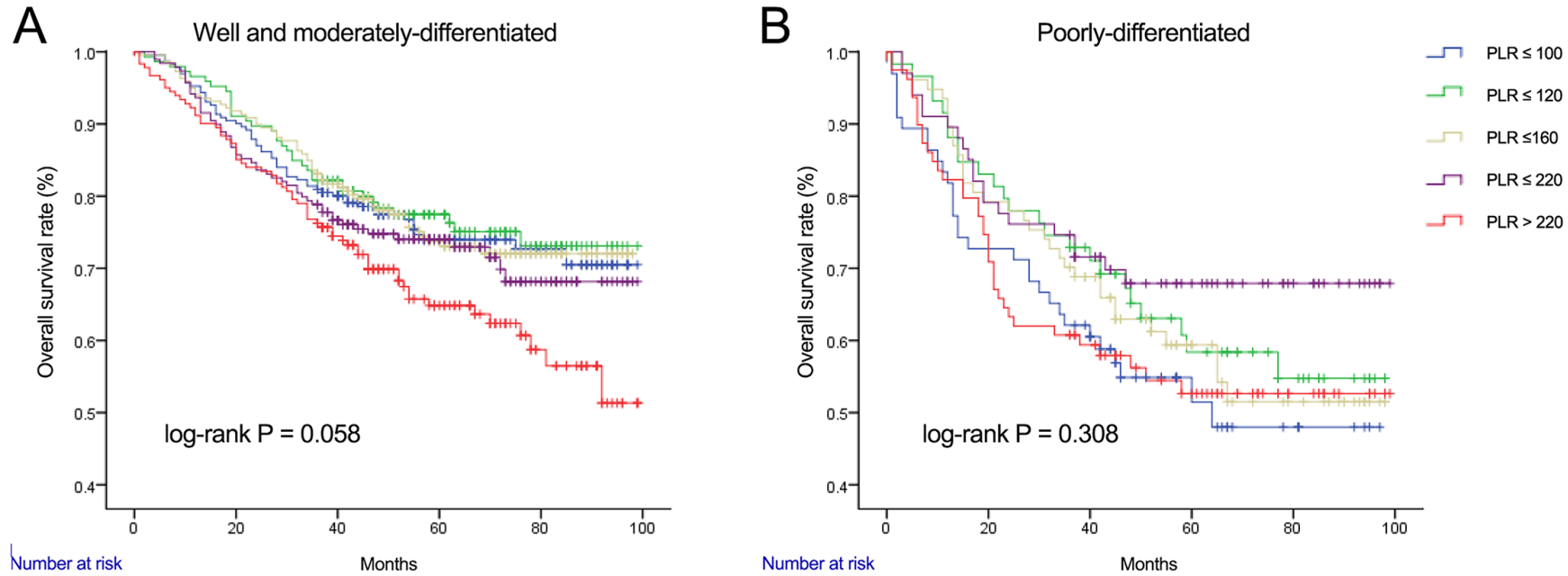

\begin{tabular}{|c|c|c|c|c|c|}
\hline \multicolumn{6}{|l|}{ PLR $\leq 100$ group } \\
\hline PLR $\leq 120$ group & 208 & 167 & 84 & 41 & 0 \\
\hline $\begin{array}{c}146 \\
P L R \leq 160 \text { group }\end{array}$ & 133 & 112 & 71 & 28 & 0 \\
\hline$P L R \leq 220$ group & 201 & 167 & 96 & 35 & 0 \\
\hline PLR $>220$ group & 162 & 132 & 76 & 25 & 0 \\
\hline 180 & 154 & 120 & 66 & 26 & 0 \\
\hline
\end{tabular}

\begin{tabular}{|c|c|c|c|c|c|}
\hline \multicolumn{6}{|l|}{ PLR $\leq 100$ group } \\
\hline$P L R \leq 120$ group & 48 & 35 & 15 & 7 & 0 \\
\hline $\begin{array}{c}59 \\
\mathrm{PLR} \leq 160 \text { group }\end{array}$ & 49 & 39 & 25 & 13 & 0 \\
\hline $\begin{array}{c}76 \\
P L R \leq 220 \text { group }\end{array}$ & 61 & 49 & 24 & 14 & 0 \\
\hline $\begin{array}{c}67 \\
\text { PLR }>220 \text { group }\end{array}$ & 53 & 44 & 26 & 13 & \\
\hline 78 & 56 & 43 & 27 & 11 & 0 \\
\hline
\end{tabular}

Figure 7: Overall survival of CRC patients stratified by quintiles of PLR according to well and moderate differentiation (A) and poor differentiation (B). 


\section{DISCUSSION}

In this study, a higher preoperative PLR was significantly and independently associated with higher mortality in patients with CRC $(P=0.002)$, with evidence that a cut off value could be derived from the selected. Similarly, the PLR showed close association with DFS, although not significant $(P=0.078)$. The results remained robust when using different cut off values and on analysis of the subgroups stratified by clinic-pathological factors, especially when comparing the highest quintile of PLR with the lowest quintile. The PLR showed a close relationship with not only tumor related characteristics, such as tumor stage and the serum level of CEA, but also nutritional status, such as total protein and BMI, which also reflected cachexia due to hypercytokinemia resulting from tumor progression [19]. Among the CRC patients stratified by clinical-pathological factors, the results from multivariate analysis indicated that the PLR was associated with OS, along with tumor-related factors, such as tumor stage, the presence of vascular invasion and CEA. It could be concluded, therefore, that the PLR have potential utility in predicting the mortality of patients with CRC.

Recent studies have demonstrated that the elevated PLR has a relationship with adverse postoperative survival in patients with several types of cancer, such as pancreatic [20], ovarian [21], gastric [22], prostate [23], esophagus [24] and colorectal cancer [13-15]. Previously, two meta-analyses of the patients with solid tumors have demonstrated that a high PLR was associated with a worse OS in various solid tumors including CRC $[25,26]$. Although current evidence supporting a role for the PLR as a prognostic indicator for patients with CRC has been relatively sparse and the power of predictive survivability less than other inflammation-based scores, such as Glasgow Prognostic Score and value of neutrophil-to-lymphocyte ratio $[16,18,27,28]$, our results provide compelling evidence that the PLR is also an adverse prognostic predictors in CRC.

In the most circumstances, the optimal cut-off values for prognostic factors are selected by validating previously established cut-off values from other clinical studies. When analyzing the cut-off values of PLR, previous studies have mainly used dichotomous categorization $[13,15,18,29]$ and trichotomy cut-off $[14,27]$ and we therefore further performed sensitivity analyses by using dichotomous categorization. The results indicated that a cut-off of value of 220 for PLR, which was also the cut-off value for CRC patients in the highest quintile in this study, was able to discriminate between those with a higher risk of mortality and those with a lower risk, replicating the same findings as the risk of recurrence of the disease.

Although most of the previous studies showed that PLR had higher HRs for mortality than the observed HR in current study [13-15], some recent studies did not find an association between PLR and OS, or DFS $[16,30]$.
One recent study which utilized a cut-off value of 300 [18], has shown that high PLR was not associated with worse OS and DFS in multivariable models, which is in agreement with our findings. One possible explanation is that the higher the cut-off value chosen, the smaller numbers of patients are enrolled in clinical practice and in the current study, there were only $8.1 \%$ of patients in the PLR group of greater than 300. In the subgroup analyses of the PLR, the impact of PLR on OS was not significantly associated with poor survival by age $(\leq 65)$ and sex and one possible explanation is that the mean 59.6 months period of follow-up was not long enough to show a statistically significant effect. Another explanation for the results from the subgroup specified by age, is that the CRC patients aged $\leq 65$ would have longer life expectancy than patients aged $<65$ after surgical treatment. According to recent cancer statistics in China, male CRC patients had a higher estimated mortality than females [31] and 5-year overall and a multivariate proportional hazard model [32] showed that cancer-specific survival of CRC was significantly higher in women than in men. This may suggest that longer follow-up periods may be required to see significant outcomes in both women and men.

The specific mechanisms by which the PLR influences the prognosis for patients with CRC remains incompletely understood. It is well known that malignant solid tumors commonly induce a hypercoagulable state, which may gradually lead to thrombocytosis $[33,34]$ and this has been considered a negative prognostic factor for patients with solid cancers [35]. The proinflammatory cytokine IL-6 has an important role in the onset of reactive thrombocytosis [36]. Similarly, IL-6 has a cell-proliferative effect, promoting the differentiation of megakaryocytes to platelets in the bone marrow [37]. Peripheral platelets, lymphocytes or their ratio are thought to be indicators of the inflammatory process induced by cancer cells and a high level of platelets may promote tumor growth by increasing angiogenesis through production of vascular endothelial growth factor which has been shown to be associated with disease prognosis in patients with various cancers [38]. Lymphocytes, however, play a vital role in cancer immune surveillance and suppress a tumor maturation [39] and a decreased concentration of intratumoral $\mathrm{CD} 8^{+}$cytotoxic lymphocytes has been strongly associated with disease prognosis in patients with colorectal cancer [40].

In summary, our current study suggests that the preoperative PLR is an independent prognostic factor in patients with CRC. Future studies are required to further validate the prognostic and predictive values of PLR.

\section{METHODS}

\section{Patients}

A total of 1314 patients who underwent surgical resection for colorectal adenocarcinoma between April 
2005 and April 2011 at the First Affiliated Hospital of Wenzhou Medical University, were enrolled in this study. Patients who had clinical evidence of infection, hematological disease, enterobrosis, intestinal obstruction and received neoadjuvant therapy were excluded. All patients exceeded 18 years of age. The study was approved by the Ethics Committee of the First Affiliated Hospital of Wenzhou Medical University and written informed consents were obtained from every patient.

\section{Clinical-pathological and laboratory data}

Demographic, preoperative laboratory and pathologic data of all patients were collected from electronic medical records and reviewed. Detailed clinical data was conducted within 2 weeks before operation. Preoperative blood values including white cell, neutrophil, lymphocyte, monocyte and platelet counts were collected from a routine blood test before surgical operation. PLR was calculated as the absolute platelet count divided by absolute lymphocyte count and was determined using a Hitachi 7600 chemistry analyzer (Hitachi, Tokyo, Japan) with the kinetic method. Body mass index (BMI) was calculated as weight in kilograms divided by height in meters squared $\left(\mathrm{kg} / \mathrm{m}^{2}\right)$. Subjects were defined as obese if BMI was greater than or equal to $25 \mathrm{~kg} / \mathrm{m}^{2}$.

Patients with CRC were treated primarily by surgical resection with adjuvant chemotherapy for node-positive patients and node-negative patients with adverse pathological features according to the National Comprehensive Cancer Network guidelines. Tumor staging of CRC was performed according to the sixth edition of the American Joint Committee on Cancer staging manual. Information regarding tumor location, TNM staging and histological differentiation of tumors and vascular invasion was collected from pathological and colonoscopic sample analyses.

\section{Follow-up data}

Patients were followed up in a post-operative outpatient schedule every 3-6 months for 2 years, every 6 months thereafter for a total of 5 years and every 1 year thereafter. Colonoscopy and computed tomography (CT) were obtained at post-operative follow-up appointments in addition to blood analysis including carcinoembryonic antigen (CEA). Tumor recurrence such as suggested by elevated CEA, abnormal findings on colonscopy or the CT scan was defined as an earlier follow-up event. Information on death was obtained either from the patient's social security death index, outpatient medical records, or notifications from the family of the deceased. The deadline of follow-up time was June 1, 2014. OS was calculated from the date of surgery to the date of death or the date of last follow-up. Disease-free survival (DFS) was calculated as the time from the date of surgery to the time of recurrence or date of last follow-up.

\section{Statistical analysis}

Continuous variables were tested for normality by using the Kolmogorov-Smirnov test. Continuous data with a normal distribution were expressed as the mean \pm standard deviation and compared using a standard $t$ test. Otherwise, continuous data with non-normal distribution were compared using the Wilcoxon rank-sum test. Categorical variables were expressed as percentage and compared using the Chi-square test or Fisher's exact test as appropriate. Based on the distribution of PLR and the size of the study population, patients were stratified into quintiles of the PLR (quintile 1, quintile 2, quintile 3, quintile 4 , quintile 5). The demographic and clinicpathological characteristics were compared between the quintiles. The optimal cut-off levels for PLR were calculated by applying receiver operating curve analysis for the dichotomous categorization. Kaplan-Meier survival curves with log-rank tests and Cox proportional hazard regression analyses, recording patients at the time of last follow-up visit, were used to compare the OS and DFS rates. Variables with $P \leq 0.1$ in the univariate Cox regression analysis were progressed to a multivariate analysis using forward stepwise selection. All $P$ values were two sided and a $P$ value $<0.05$ was considered to be statistically significant. Statistical analysis was performed using SPSS version 19.0 software (SPSS, Chicago, IL, USA) and Med Calc version 13.0.0.0 (Med Calc Software, Mariakerke, Belgium).

\section{ACKNOWLEDGMENTS AND FUNDING}

This work was supported by grants from the National Natural Science Foundation of China (81500665, 81501823), Zhejiang Engineering Research Center of Intelligent Medicine (2016E10011) and Project of New Century 551 Talent Nurturing in Wenzhou.

\section{CONFLICTS OF INTEREST}

The authors report no declarations of interest.

\section{Authors' contributions}

You J, Liu WY, Zhu GQ, Huang ZH and Zheng MH designed the study. Zhu GQ, Wang OC, Huang GQ collected data. You J, Xie L and Guo GL did the statistical analyses. Liu WY and Zhu GQ prepared figures. You J, Xie L, Liu WY, Zhu GQ, Wang OC, Huang ZH, Guo GL, Braddock $\mathrm{M}$ and Zheng MH reviewed the results, interpreted data, and wrote the manuscript. All authors have made an intellectual contribution to the manuscript and approved the submission. 


\section{REFERENCES}

1. Brenner H, Kloor M, Pox CP. Colorectal cancer. Lancet. 2014; 383:1490-1502.

2. Siegel R, Desantis C, Jemal A. Colorectal cancer statistics. CA Cancer J Clin. 2014; 64:104-117.

3. De Angelis R, Sant M, Coleman MP, Francisci S, Baili P, Pierannunzio D, Trama A, Visser O, Brenner H, Ardanaz E, Bielska-Lasota M, Engholm G, Nennecke A, et al. Cancer survival in Europe 1999-2007 by country and age: results of EUROCARE - 5-a population-based study. Lancet Oncol. 2014; 15:23-34.

4. Hanahan D, Weinberg RA. Hallmarks of cancer: the next generation. Cell. 2011; 144:646-674.

5. Mantovani A, Allavena P, Sica A, Balkwill F. Cancer-related inflammation. Nature. 2008; 454:436-444.

6. Colotta F, Allavena P, Sica A, Garlanda C, Mantovani A. Cancer-related inflammation, the seventh hallmark of cancer: links to genetic instability. Carcinogenesis. 2009; 30:1073-1081.

7. Roxburgh CS, McMillan DC. Cancer and systemic inflammation: treat the tumour and treat the host. $\mathrm{Br} \mathrm{J}$ Cancer. 2014; 110:1409-1412.

8. Laird BJ, Kaasa S, McMillan DC, Fallon MT, Hjermstad MJ, Fayers P, Klepstad P. Prognostic factors in patients with advanced cancer: a comparison of clinicopathological factors and the development of an inflammation-based prognostic system. Clin Cancer Res. 2013; 19:5456-5464.

9. Cetinkunar S, Guzel H, Emre Gokce I, Erdem H, Gulkan S, Aktimur R, Kucuk B, Imamoglu I, Kargici H. High levels of platelet/lymphocyte ratio are associated with metastatic gastric cancer. J BUON. 2015; 20:78-83.

10. Chen Q, Dai Z, Yin D, Yang LX, Wang Z, Xiao YS, Fan J, Zhou J. Negative impact of preoperative plateletlymphocyte ratio on outcome after hepatic resection for intrahepatic cholangiocarcinoma. Medicine (Baltimore). 2015; 94:e574.

11. Feng JF, Huang Y, Chen QX. Preoperative platelet lymphocyte ratio (PLR) is superior to neutrophil lymphocyte ratio (NLR) as a predictive factor in patients with esophageal squamous cell carcinoma. World J Surg Oncol. 2014; 12:58.

12. Hong C, Wei Y, Jiang J, Zhao C, Liang G, Wang G, Yang H. Associations between lifestyles and neutrophil-lymphocyte and platelet-lymphocyte ratios in colorectal cancer. Asia-Pacific journal of clinical oncology. 2014; 10:168-174.

13. Ozawa T, Ishihara S, Nishikawa T, Tanaka T, Tanaka J, Kiyomatsu T, Hata K, Kawai K, Nozawa H, Kazama S, Yamaguchi H, Sunami E, Kitayama J, et al. The preoperative platelet to lymphocyte ratio is a prognostic marker in patients with stage II colorectal cancer. Int J Colorectal Dis. 2015; 30:1165-1171.
14. Kwon HC, Kim SH, Oh SY, Lee S, Lee JH, Choi HJ, Park KJ, Roh MS, Kim SG, Kim HJ. Clinical significance of preoperative neutrophil-lymphocyte versus plateletlymphocyte ratio in patients with operable colorectal cancer. Biomarkers. 2012; 17:216-222.

15. Szkandera J, Pichler M, Absenger G, Stotz M, Arminger F, Weissmueller M, Schaberl-Moser R, Samonigg H, Kornprat P, Stojakovic T, Avian A, Gerger A. The elevated preoperative platelet to lymphocyte ratio predicts decreased time to recurrence in colon cancer patients. Am J Surg. $2014 ; 208: 210-214$.

16. Azab B, Mohammad F, Shah N, Vonfrolio S, Lu W, Kedia S, Bloom SW. The value of the pretreatment neutrophil lymphocyte ratio vs. platelet lymphocyte ratio in predicting the long-term survival in colorectal cancer. Cancer Biomark. 2014; 14:303-312.

17. Azab B, Shah N, Radbel J, Tan P, Bhatt V, Vonfrolio S, Habeshy A, Picon A, Bloom S. Pretreatment neutrophil/ lymphocyte ratio is superior to platelet/lymphocyte ratio as a predictor of long-term mortality in breast cancer patients. Med Oncol. 2013; 30:432.

18. Choi WJ, Cleghorn MC, Jiang H, Jackson TD, Okrainec A, Quereshy FA. Preoperative Neutrophil-to-Lymphocyte Ratio is a Better Prognostic Serum Biomarker than Plateletto-Lymphocyte Ratio in Patients Undergoing Resection for Nonmetastatic Colorectal Cancer. Ann Surg Oncol. 2015; 22:603-613.

19. Wakuda R, Miki C, Kusunoki M. Autoreactivity against interleukin 6 as a risk factor in elderly patients with colorectal carcinoma. Arch Surg. 2001; 136:1274-1279.

20. Shirai Y, Shiba H, Sakamoto T, Horiuchi T, Haruki K, Fujiwara Y, Futagawa Y, Ohashi T, Yanaga K. Preoperative platelet to lymphocyte ratio predicts outcome of patients with pancreatic ductal adenocarcinoma after pancreatic resection. Surgery. 2015; 158:360-365.

21. Asher V, Lee J, Innamaa A, Bali A. Preoperative platelet lymphocyte ratio as an independent prognostic marker in ovarian cancer. Clin Transl Oncol. 2011; 13:499-503.

22. Chen XL, Xue L, Wang W, Chen HN, Zhang WH, Liu K, Chen XZ, Yang K, Zhang B, Chen ZX, Chen JP, Zhou ZG, $\mathrm{Hu}$ JK. Prognostic significance of the combination of preoperative hemoglobin, albumin, lymphocyte and platelet in patients with gastric carcinoma: a retrospective cohort study. Oncotarget. 2015; 6:41370-41382. doi: 10.18632/ oncotarget.5629.

23. Li F, Hu H, Gu S, Chen X, Sun Q. Platelet to lymphocyte ratio plays an important role in prostate cancer's diagnosis and prognosis. Int J Clin Exp Med. 2015; 8:11746-11751.

24. Feng JF, Huang Y, Zhao Q, Chen QX. Clinical significance of preoperative neutrophil lymphocyte ratio versus platelet lymphocyte ratio in patients with small cell carcinoma of the esophagus. ScientificWorld Journal. 2013; 2013:504365.

25. Templeton AJ, Ace O, McNamara MG, Al-Mubarak M, Vera-Badillo FE, Hermanns T, Seruga B, Ocana A, 
Tannock IF, Amir E. Prognostic role of platelet to lymphocyte ratio in solid tumors: a systematic review and meta-analysis. Cancer Epidemiol Biomarkers Prev. 2014; 23:1204-1212.

26. Zhou X, Du Y, Huang Z, Xu J, Qiu T, Wang J, Wang T, Zhu W, Liu P. Prognostic value of PLR in various cancers: a meta-analysis. PloS one. 2014; 9:e101119.

27. He W, Yin C, Guo G, Jiang C, Wang F, Qiu H, Chen X, Rong R, Zhang B, Xia L. Initial neutrophil lymphocyte ratio is superior to platelet lymphocyte ratio as an adverse prognostic and predictive factor in metastatic colorectal cancer. Med Oncol. 2013; 30:439.

28. Choi KW, Hong SW, Chang YG, Lee WY, Lee B, Paik IW, Lee H. Inflammation-based score (Glasgow prognostic score) as an independent prognostic factor in colorectal cancer patients. Ann Surg Treat Res. 2014; 86:309-313.

29. Carruthers R, Tho LM, Brown J, Kakumanu S, McCartney E, McDonald AC. Systemic inflammatory response is a predictor of outcome in patients undergoing preoperative chemoradiation for locally advanced rectal cancer. Colorectal Dis. 2012; 14:e701-707.

30. Baranyai Z, Krzystanek M, Josa V, Dede K, Agoston E, Szasz AM, Sinko D, Szarvas V, Salamon F, Eklund AC, Szallasi Z, Jakab F. The comparison of thrombocytosis and platelet-lymphocyte ratio as potential prognostic markers in colorectal cancer. Thromb Haemost. 2014; 111:483-490.

31. Chen W, Zheng R, Baade PD, Zhang S, Zeng H, Bray F, Jemal A, Yu XQ, He J. Cancer statistics in China, 2015. CA Cancer J Clin. 2016; 66:115-132.

32. Chou CL, Weng SF, Lin JK, Chang SC. Role for gender in colorectal cancer risk: a Taiwan population-based study. Int J Colorectal Dis. 2013; 28:1001-1008.
33. Ishizuka M, Nagata H, Takagi K, Iwasaki Y, Kubota K. Preoperative thrombocytosis is associated with survival after surgery for colorectal cancer. J Surg Oncol. 2012; 106:887-891.

34. Monreal M, Fernandez-Llamazares J, Pinol M, Julian JF, Broggi M, Escola D, Abad A. Platelet count and survival in patients with colorectal cancer-a preliminary study. Thromb Haemost. 1998; 79:916-918.

35. Sasaki K, Kawai K, Tsuno NH, Sunami E, Kitayama J. Impact of preoperative thrombocytosis on the survival of patients with primary colorectal cancer. World J Surg. 2012; 36:192-200.

36. Sierko E, Wojtukiewicz MZ. Platelets and angiogenesis in malignancy. Semin Thromb Hemost. 2004; 30:95-108.

37. Ruscetti FW. Hematologic effects of interleukin-1 and interleukin-6. Curr Opin Hematol. 1994; 1:210-215.

38. Wiesner T, Bugl S, Mayer F, Hartmann JT, Kopp HG. Differential changes in platelet VEGF, Tsp, CXCL12, and CXCL4 in patients with metastatic cancer. Clin Exp Metastasis. 2010; 27:141-149.

39. Dunn GP, Old LJ, Schreiber RD. The immunobiology of cancer immunosurveillance and immunoediting. Immunity. 2004; 21:137-148.

40. Daster S, Eppenberger-Castori S, Hirt C, Soysal SD, Delko T, Nebiker CA, Weixler B, Amicarella F, Iezzi G, Governa V, Padovan E, Mele V, Sconocchia G, et al. Absence of myeloperoxidase and CD8 positive cells in colorectal cancer infiltrates identifies patients with severe prognosis. Oncoimmunology. 2015; 4:e1050574. 